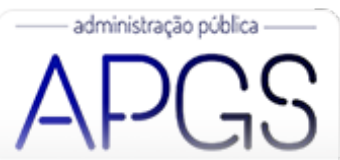

Administração Pública e Gestão Social ISSN: 2175-5787

apgs@ufv.br

Universidade Federal de Viçosa

Brasil

\title{
Corrupção no Setor Público: Agenda de Pesquisa e Principais Debates a Partir da Literatura Internacional
}

Barreto de Souza, Fábio Jacinto; de Almeida Midlej e Silva, Suylan; de Oliveira Gomes, Adalmir Corrupção no Setor Público: Agenda de Pesquisa e Principais Debates a Partir da Literatura Internacional Administração Pública e Gestão Social, vol. 11, núm. 3, 2019

Universidade Federal de Viçosa, Brasil

Disponível em: http://www.redalyc.org/articulo.oa?id=351559268001

Esta obra está bajo una Licencia Creative Commons Atribución-NoComercial-SinDerivar 3.0 Internacional 


\title{
Corrupção no Setor Público: Agenda de Pesquisa e Principais Debates a Partir da Literatura Internacional
}

\author{
Public sector corruption: Research Agenda and Debates from international literature \\ Corrupción del sector público: la agenda de investigación y los principales debates de la literatura internacional
}

Fábio Jacinto Barreto de Souza

Redalyc: http://www.redalyc.org/articulo.oa?

Universidade de Brasilia, Brasil

id $=351559268001$

fabiojjsouza@gmail.com

Suylan de Almeida Midlej e Silva

Universidade de Brasilia, Brasil

suylan@unb.br

Adalmir de Oliveira Gomes

Universidade de Brasilia, Brasil

adalmirdeoliveira@gmail.com

Recepção: 27 Novembro 2017

Aprovação: 15 Julho 2018

Publicado: 01 Julho 2019

\section{Resumo:}

Este artigo pretende identificar e discutir como a agenda de pesquisa sobre a corrupção no setor público vem sendo abordada pela literatura internacional e quais são as questões sobre o fenômeno que estão no centro do debate acadêmico. Para isso, foram coletados artigos em duas bases de indexação de artigos científicos (Scopus e Web of Science). Ao todo foram analisadas 211 produções internacionais, entre artigos teóricos e empíricos que tratam da corrupção. Os resultados apontam para um campo de estudo em crescimento, com uma variedade de métodos e técnicas de pesquisa e com cinco temas centrais (conceito, causas, consequências, formas de se medir e de se combater a corrupção) que permeiam todos os artigos estudados. Ao final, o que se conclui é que ainda existe uma considerável agenda de pesquisa sobre o tema e sobre os cinco tópicos identificados.

Palavras-chave: Administração Pública, Corrupção no Setor Público, Revisão Sistemática.

\section{Abstract:}

This article aims to identify and discuss a research agenda on corruption in the public sector has been approached in international literature and how are these themes being addressed in the academia. For this study, we collected articles in two databases of scientific articles (Scopus and Web of Science). 211 international productions were analyzed among articles that deal with the clinical and empirical cover corruption issues. The results point to a growing field of study, with a variety of research methods and techniques. In the end, we concluded that there's still a considerable research agenda on the topic.

KeYwords: Public Administration, Corruption in the Public Sector, Systematic Review.

\section{RESUMEN:}

Este estudio pretende identificar y discutir cómo la agenda de investigación sobre la corrupción en el sector público viene siendo abordada por la literatura internacional y cuáles son las cuestiones sobre el fenómeno que están en el centro del debate académico. Para esto, se recogieron artículos en dos bases de identificación de artículos científicos (Scopus y Web of Science). En total se analizaron 211 producciones internacionales entre artículos teóricos y empíricos que tratan de la corrupción. Los resultados apuntan a un campo de estudio en crecimiento, con una variedad de métodos y técnicas de investigación y con cinco temas centrales (concepto, causas, consecuencias, formas de medir y de combatir la corrupción), que permean todos los artículos estudiados . Al final lo que se concluye es todavía existe una considerable agenda de investigación sobre el tema y sobre los cinco tópicos identificados.

Palabras clave: Administración Pública, Corrupción en el Sector Público, Revisión Sistemática. 


\section{INTRODUÇÃo}

A corrupção pode significar diferentes coisas em diferentes contextos. No caso de uma concepção política, ela pode ser representada por ganhos ilícitos relacionados ao poder político ou à manutenção desse poder. Já em um contexto econômico, os ganhos podem estar relacionados a ativos financeiros. A corrupção também pode ser dividida em corrupção privada, que é quando um agente privado "compra” outros agentes privados para ter acesso a recursos escassos ou derrubar concorrentes, ou quando pessoas compram ingressos de um cambista para não terem de enfrentar uma fila. Já no contexto da Administração Pública, na maior parte dos casos, a corrupção está relacionada ao uso de cargos públicos para ganhos privados, em que um agente público se encarrega de realizar algum tipo de tarefa utilizando de má-fé para enriquecimento privado. O presente estudo trata especificamente desse segundo tipo de corrupção, que ocorre na esfera pública.

A corrupção no setor público está presente em todos os países, desde os países desenvolvidos até os países mais pobres do mundo, como será evidenciado neste estudo (Erlingsson, Bergh, \& Sjölin, 2008). Embora a literatura aponte para uma predominância do fenômeno em países pobres, sua incidência pode ser igualmente danosa em países considerados ricos e em desenvolvimento. A desigualdade social, a evasão de receita, a desaceleração do crescimento econômico e a consequente falta de recursos para investimentos estão entre as consequências mais comuns da corrupção, registradas na literatura relacionada ao tema.

Diversos estudos associam a corrupção à evasão de receitas vindas de taxas tributárias (Joshi \& Ayee, 2009; Rosenbloom \& Gong, 2013; Harbers, 2015; Gong \& Zhou, 2015). Os sistemas tributários da maioria dos países pobres são caracterizados por ampla corrupção e consequente evasão de receitas tributárias (Fjeldstad, 2003). Uma dificuldade para se compreender melhor essa relação é que, segundo Fjeldstad (2003, p. 167), informações confiáveis sobre corrupção e taxas de evasão são "obviamente" difíceis de conseguir. A evasão fiscal na Itália em 2012 chegou à soma de 275 bilhões de euros (Esposito \& Ricci, 2015). Como observado, em países onde a carga tributária é alta e o retorno social das ações governamentais e políticas públicas é percebido como baixo, a corrupção tende a prosperar. $\mathrm{Na}$ Ucrânia, as altas taxas de impostos fizeram com que os empregadores acabassem pagando aos fiscais uma taxa para evitar um montante real de impostos devidos à corrupção (Peacock \& Cordner, 2016).

A Commission on the Causes of Corruption in Tanzania identificou quatro grandes causas para corrupção fiscal naquele país: A intervenção política de grupos de pressão e organizações; altas taxas de impostos, como no caso da Ucrânia; uma complicada política de regulação tributária e baixos salários dos servidores públicos envolvidos nessas questões (Fjeldstad, 2003). Um caso emblemático no Brasil ocorreu no Conselho de Ações e Recursos Fiscais (CARF), do Ministério da Fazenda: O conselho opera como um tribunal responsável por julgar em instância administrativa recursos referentes a questões fiscais. Em 2015 a Polícia Federal deflagrou a Operação Zelotes, na qual foram indiciados por corrupção diversos membros do conselho, inclusive um ex-presidente, sob a acusação de vender decisões reduzindo a alíquota de impostos para grandes grupos econômicos. O prejuízo estimado é de $\mathrm{R} \$ 19$ bilhões em evasão de receitas.

Diversos problemas permeiam o campo de estudo da corrupção, como, por exemplo, tributação, zonas de fronteira, policiais, membros do alto escalão, licitações e contratos, obras públicas e campanhas eleitorais. Essa diversidade de temas oferece um amplo campo de pesquisa a respeito do fenômeno. Trata-se de um problema complexo e de uma literatura que precisa se moldar ao tempo, uma vez que há evidências de que a corrupção vem se transformando juntamente com a administração pública e a sociedade (Rose-Ackerman, 2002; Klitgaard, 1994). Temas contemporâneos como a governança participativa já começam a ser estudados como mecanismos que, se por um lado ampliam a participação democrática, por outro podem abrir espaço para a corrupção. As organizações que operam em forma de redes, que antes eram utilizadas por grupos de tráfico de drogas e terroristas, passaram a ser utilizadas também para lidar com esquemas intercontinentais de corrupção (Klitgaard, 1994). Além da complexidade e variedade de temas que permeiam esse tema, algumas questões parecem ser centrais para o estudo do tema. Uma grande quantidade de autores está reunindo esforços no 
sentido de se avançar na compreensão do fenômeno e, embora exista uma ampla literatura internacional sobre o tema, muitas questões parecem ainda não estar claras e pacificadas na literatura especializada, tais como o próprio conceito de corrupção, formas de medi-la, suas causas e suas consequências. Nesse sentido, este estudo tem como objetivo central identificar e discutir como vem sendo direcionada a agenda de pesquisa sobre a corrupção no setor público, na literatura internacional em inglês, e quais são as questões centrais discutidas nessas pesquisas.

Por uma questão de ordem, o presente artigo foi estruturado em quatro seções. Além desta introdução, há uma seção tratando dos aspectos metodológicos da pesquisa, outra seção apresentando os resultados e a discussão sobre os resultados da pesquisa. A seção que trata dos resultados, além de apresentar uma análise descritiva da produção pesquisada, apresenta como se chegou aos tópicos centrais que direcionaram a formulação das questões. As outras subseções tratam consecutivamente dos resultados sobre cada questão elaborada. Por fim, há uma seção de conclusão e considerações finais.

\section{Metodologia}

A pesquisa foi dividida em duas fases, uma primeira fase exploratória sobre a amostra bibliográfica e outra fase que buscou identificar quais são os temas centrais e como eles são abordados em cada artigo. A primeira fase teve como objetivo identificar quem são os autores, universidade de origem, volume de produção no tempo, qualidade da produção (fator JCR), países mais citados nessas produções, tipo de estudo (empírico ou teórico), abordagem (qualitativa ou quantitativa), métodos e instrumentos de pesquisa utilizados nessa literatura. Já na segunda fase, buscou-se elaborar uma síntese preliminar do tema por meio da busca exploratória de estudos relacionados com o objeto de pesquisa, desenvolvimento de uma síntese preliminar do material por meio de descrição textual, exploração das relações entre os estudos, panorama da produção a respeito dos temas centrais e reflexão crítica e síntese narrativa da literatura estudada.

Operacionalmente, a coleta de dados objetivou encontrar artigos que tratavam de corrupção no setor público. Para a coleta dos dados, foram utilizadas duas bases de indexação de periódicos: Scopus e Web of Science. Não foram utilizadas outras bases de dados, uma vez que se observou que a quantidade de resultados, além de muito inferior, se repetia. Os critérios de busca foram definidos da seguinte forma: conter no título algum termo com o prefixo "corrup", porém com restrição nos campos (subject) de conhecimento do tema Public Administration. Não houve nenhum critério de exclusão referente à data de publicação dos periódicos. A busca por títulos retornou 125 artigos na base Web of Science e 97 artigos na base Scopus. Embora não tenha havido delimitação de busca quanto ao período de publicação dos artigos, observou-se que o primeiro artigo data do ano de 1989 e o último, de 2017.

Os artigos foram extraídos em sua íntegra e organizados em um repositório. Os registros duplicados foram removidos e os dados inconsistentes e ausentes foram corrigidos por procedimento manual. Após todos os procedimentos, a amostra final foi composta por 211 artigos. Também se extraiu dos resultados da busca os metadados dos artigos pesquisados disponíveis para download na própria base indexadora.

Os dados foram estruturados em duas bases de dados, a primeira base (B1) está relacionada à primeira fase da pesquisa e foi construída a partir dos metadados extraídos dos próprios artigos, das bases de dados pesquisadas (Scopus e WoS - Web of Science). Acrescentou-se a essa base (B1) os dados do fator JCR (Journal Citation Reports) de cada periódico. A segunda base de dados foi elaborada com objetivo de dar suporte à segunda fase da pesquisa e continha os artigos em sua íntegra. Nesse caso, os artigos foram organizados e estruturados em uma base de dados para uso do software Atlas TI v. 7.5.

A análise qualitativa dos dados foi realizada com suporte do referido software. $\mathrm{O}$ tipo de análise de dados pode ser considerado como Computer-Assisted Qualitative Data Analysis Software (CAQDAS). Para tanto, foram criadas famílias de códigos identificadas na leitura exploratória dos textos, que como produto denominou-se nesse estudo de "temas centrais". Esses códigos foram associados aos trechos de 
texto que continham os assuntos relacionados ao tópico. $\mathrm{O}$ software teve papel importante em marcar os trechos, associar aos códigos e gerar os relatórios para que fossem feitas as análises. A partir das análises foram feitas uma reflexão crítica e uma síntese do material encontrado. Especificamente utilizou-se a técnica CAQDAS proposta por Friese (2014), denominada NCT (Notice Things, Collecting Things, Trinking about Things). A técnica parte de uma leitura inicial onde são tomadas notas sobre achados (no software) e, assim, começa-se a criar códigos semânticos também em sua plataforma. Com o avanço da pesquisa, os códigos são relacionados a partes/marcações nos artigos e vão sendo refinados e consolidados. Por exemplo, se de início são elaborados três códigos a partir da leitura: a. Falta de Regulamentação, b. Fragilidade Institucional, c. Baixa Remuneração, ao final, esses códigos podem ser associados a um outro código chamado "Causas da Corrupção". No início os códigos não desempenham um papel de análise apenas de organização mas, com a sequência da pesquisa e marcações, os códigos vão ganhando significado. Em seguida as "coisas" encontradas são filtradas, e organizadas a partir dos códigos (Collecting Things), depois inicia-se o processo de geração de relatórios a partir do software e análise por parte do pesquisador (Thinking about Things). Segundo a autora, o método pode ser utilizado sempre que se precisa descobrir padrões, processos, sequências ou tipologias. Ao final da pesquisa, a estrutura de análise qualitativa foi formada por seis códigos ou famílias de códigos com os temas centrais.

\section{Resultados da Pesquisa}

As seções seguintes foram construídas de forma a apresentar um panorama da literatura pesquisada e, em seguida, abordar os resultados e discussão de cada tópico direcionador. Como observado anteriormente, foram identificados cinco grandes tópicos nos quais o tema Corrupção na Administração Pública é debatido: (a) conceito de corrupção, (b) causas da corrupção, (c) consequências da corrupção, (d) medidas de corrupção, e (e) prevenção e combate à corrupção. Nesse sentido, a revisão da literatura observará essa sequência lógica para se discutir o tema. Obviamente não se busca esgotar o tema ou cobrir todo o conteúdo, mas sim situar o leitor em relação ao que vem sendo discutido sobre corrupção na Administração Pública.

\subsection{Análise Descritiva da Literatura}

Considerando toda a amostra de pesquisa, ao todo foram encontrados registros de produção em 63 países. Dos 211 artigos, 35 são de autores que atuam em instituições americanas, 20 de autores da China, 18 da Austrália, 16 da Inglaterra e 15 de autores de instituições da Rússia, Canadá e Escócia. Entre as universidades, foi observada concentração significativa de estudos apenas na China, onde 50\% (10 artigos) da amostra daquele país foi produzida pela Universidade da Cidade de Hong Kong, sendo seis deles do mesmo autor (Gong T. , 2011; Gong T. , 2009; Gong \& Wu, 2012; Gong \& Wu, 2012; Gong \& Zhou, 2015; Gong, Wang, \& Ren, 2015)

Quanto ao volume de produção, é possível identificar um crescente aumento ao longo do tempo, sobretudo quando considerados os primeiros anos, como se observa na Figura 1. É possível notar que a partir de 2008 houve um aumento considerável na quantidade de publicações, passando de 9 em 2007 para 25 em 2012. Isso indica que o tema tem atraído cada vez mais a atenção de pesquisadores, do modo que é possível esperar o aumento dos estudos nos próximos anos. 


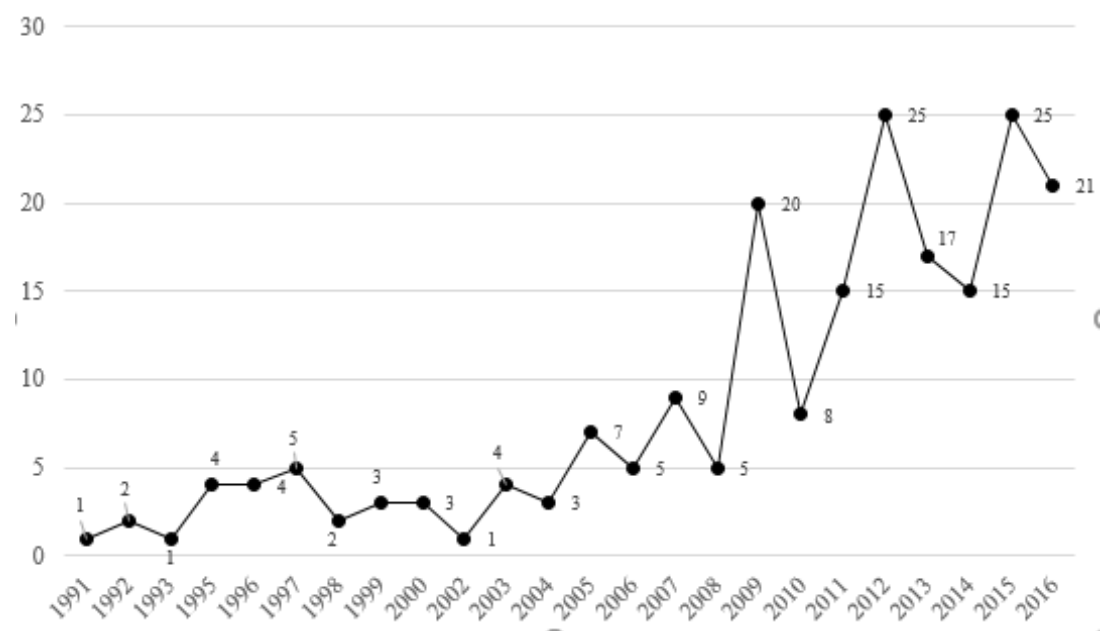

Figura 1 - Evolução da produção acadêmica sobre o tema.

No que se refere à qualidade acadêmica da produção a respeito da corrupção na Administração Pública, considerando o fator de impacto (JRC) dos artigos, a Tabela 1 detalha os resultados encontrados. Grande parte da produção encontrada possui fator de impacto entre 0,02 e 0,94, sendo que apenas 17 artigos apresentavam fator equivalente a zero. Quanto aos periódicos, foram encontradas concentrações em apenas dois: Internacional Journal of Policy Administration and Institutions ( $n=12$; JCR=3,42), e Public Administration Review ( $\mathrm{n}=10$; JCR $=2,64)$.

Tabela 1 - Qualidade da produção acadêmica revisada, segundo o fator de impacto (JCR)

\begin{tabular}{llll}
\hline $\begin{array}{l}\text { Faixa (Fator } \\
\text { JCR) }\end{array}$ & $\begin{array}{l}\text { Média } \\
\text { (JCR) }\end{array}$ & Desvio (s) & $\begin{array}{l}\text { Quantidade } \\
\text { de Artigos }\end{array}$ \\
\hline $2,64-3,89$ & 3,02 & 0,39 & 26 \\
\hline $1,07-1,98$ & 1,43 & 0,32 & 32 \\
\hline $0,02-0,94$ & 0,68 & 0,24 & 136 \\
\hline 0 & - & - & 17
\end{tabular}

Com base nos aspectos regionais, a análise quantitativa buscou, ainda, identificar os países que tiveram mais citações dentro da amostra, considerando para isso a quantidade de incidências por artigo, ou seja, se o país foi muito citado e se esse número de citações está concentrado em poucos ou muitos artigos. A Figura 2 mostra a dispersão entre a quantidade de citações (eixo X) e a quantidade de incidências (eixo Y). Como se observa, a China $(1.148 ; 63)$, embora seja um país muito citado, há uma concentração dessas citações em uma quantidade relativamente pequena de periódicos, quando observada a amostra total do estudo (211 artigos). Por outro lado, os Estados Unidos (391; 97) são citados em diversos artigos. Austrália, Índia, Rússia, Alemanha, Singapura, Japão, França e Uganda também são países com bastante incidência de relatos. 


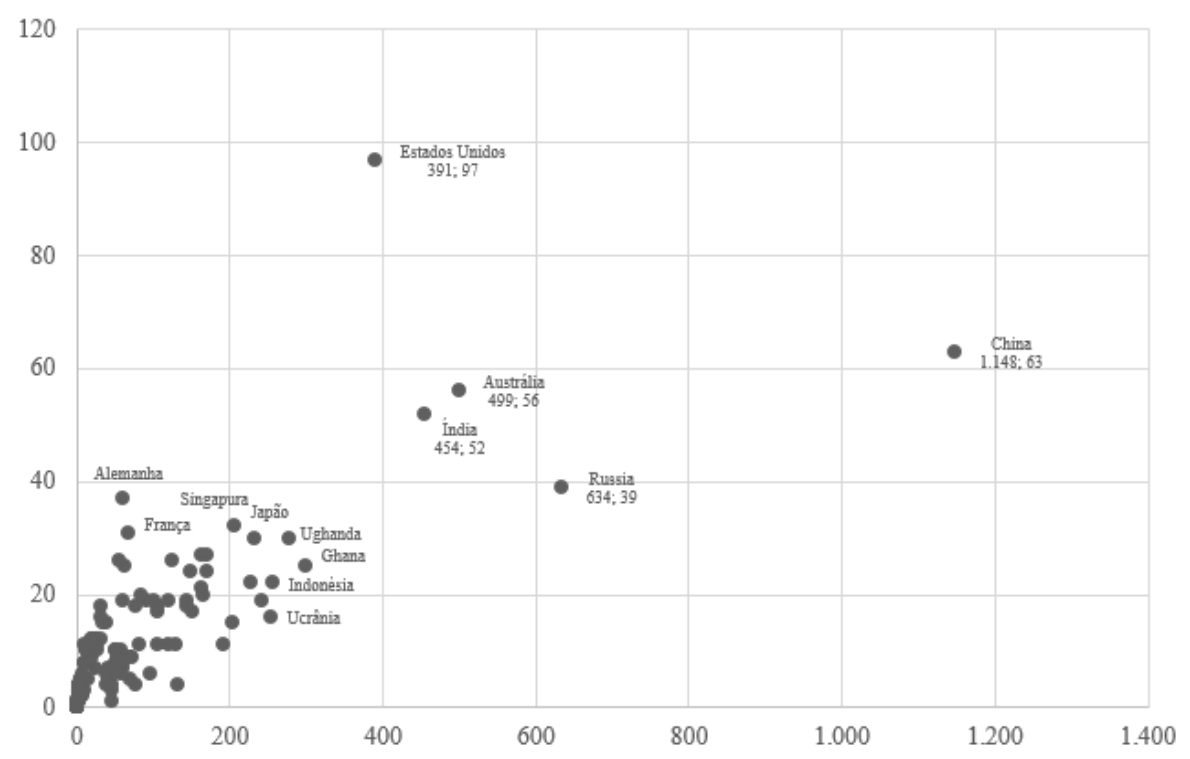

Figura 2 - Citações por ocorrências

A análise inicial permite questionar os motivos pelos quais esses países são tão citados. No caso da China, 14 artigos tratam especificamente daquele país (Gong T. , 2009; Li, Xiao, \& Gong, 2015; Deng, Wang, Zhang, Huang, \& Cui, 2014; Ke, Wang, \& Chan, 2013; Rosenbloom \& Gong, 2013; Gong T., 2011; Gong \& Zhou, 2015; Chang \& Kerr, 2017) (Chan \& Ma, 2011; Yang, 2009; Gong, Wang, \& Ren, 2015; Tsao \& Worthley, 1996; Cheung, 2012; Lin, 1995; Zhu \& Jiao, 2012). Dentre esses estudos, onze tratam principalmente de quais mecanismos o país tem adotado para lidar com a corrupção. Estudos de caso a respeito da situação peculiar de Hong Kong também são incidentes na amostra, com artigos que tratam, em sua maioria, de experiências de combate à corrupção.

Ao contrário do que foi observado na China, apenas três artigos tratam especificamente do fenômeno da corrupção nos Estados Unidos. O artigo de Johnston et. al. (2012) aborda medidas para controlar a corrupção nos Estados Unidos, enquanto o artigo de Dincer e Gunalp, (2012) trata dos efeitos da corrupção, sobretudo considerando a desigualdade. Por sua vez, o artigo de Cordis e Milyo (2016) traz uma crítica às fontes de dados disponibilizadas pelo Governo Americano, que dificultam estudos e a transparência no combate à corrupção.

Quanto ao tipo de pesquisa, há predominância de estudos empíricos, com 128 ocorrências, enquanto 83 artigos são teóricos. Com relação aos trabalhos empíricos, foram encontrados $42 \mathrm{com}$ abordagem quantitativa, 55 com abordagem qualitativa e 9 artigos com abordagem quali-quanti. Os estudos qualitativos, em sua maioria, utilizaram entrevistas (39), grupo focal (17) e pesquisa documental. As entrevistas foram conduzidas com agentes públicos; investigadores; burocratas de rua, como policiais, e até com integrantes do alto escalão dos governos, além de cidadãos e agentes privados, tais como os empresários (Peacock \& Cordner, 2016; Zhu \& Jiao, 2012; Lin, 1995; Kopytko, 2016; Chen, Li, \& Wang, 2010; Gong \& Zhou, 2015) O estudo de Jancsics e Jávór (2012), por exemplo, realizou 45 entrevistas com membros de diversas organizações de Budapeste, na Hungria, que estiveram envolvidos em esquemas de corrupção. $\mathrm{O}$ estudo de Škrbec \& Dobovšek (2013) envolveu uma série de agentes públicos, sendo que, ao todo, foram conduzidas 45 entrevistas com diferentes agentes envolvidos com o tema corrupção na Eslovênia: policiais, juízes, promotores de justiça, professores e pesquisadores, representantes de agências de combate à corrupção, jornalistas e membros de órgãos de controle do governo.

Entre os métodos envolvendo análise qualitativa, destaca-se a técnica de grupo focal, utilizada em pesquisas exploratórias em diversas localidades, mas sem necessariamente realizar uma análise comparativa (Forgia, Raha, Shaik, Maheshwari, \& Ali, 2015; Grimes \& Wangnerud, 2010; Gong \& Zhou, 2015; Walton, 2013; Grodeland, Koshechkina, \& Miller, 1997). Quando o objetivo era identificar a percepção dos cidadãos 
e/ou de determinada comunidade, ou realizar estudos comparativos, os pesquisadores fizeram opção pelo survey. O recurso foi utilizado em 75 estudos. Quando o método não foi aplicado empiricamente (survey methodology), os autores utilizaram dados de outros surveys. Os dados da Transparência Internacional (Corruption Perceptions Index - CPI), por exemplo, foram utilizados em 40 artigos (Zhu \& Jiao, 2012; Yeboah-Assiamah, Asamoah, Bawole, \& Musah-Surugu, 2016; Walker, Brewer, Bozeman, Moon, \& Wu, 2013; Gong T. , 2011; Villoria, Ryzin, \& Lavena, 2013). Os estudos envolvendo surveys apresentam características de análise quantitativa e o uso de estatística inferencial e multivariada (Gong, Wang, \& Ren, 2015; Villoria, Ryzin, \& Lavena, 2013; Kim, 2010).

Ainda quanto ao uso do grupo focal, Grodeland, Koshechkina e Miller (1997), por exemplo, conduziram um estudo com a realização de 26 grupos focais em quatro países diferentes: Ucrânia, Bulgária, Eslováquia e República Checa. Os autores buscaram identificar os casos e, na visão dos membros do grupo, o que pode ser feito para prevenir e combater a corrupção. A pesquisa envolveu pessoas com alta e baixa educação, minorias étnicas, moradores de vilas, pequenas cidades e cidades de médio porte. Em Papua Nova Guiné, no estudo realizado por Walton (2013), foram conduzidos 16 grupos focais em quatro províncias, sendo que em cada província foram visitadas duas vilas. O objetivo foi identificar as consequências da corrupção em nível local e, para isso, o autor selecionou em cada província uma vila com difícil acesso a serviços públicos e outra com projetos de desenvolvimento em andamento. Para formação do grupo focal, o autor selecionou pessoas com diferentes níveis de educação de diferentes idades e de ambos os sexos.

A pesquisa documental também é um método bastante utilizado, com a instrumentação de informações provenientes de relatórios de agências, documentos oficiais do governo, leis e regulamentos, documentos policiais e reportagens jornalísticas. A pesquisa documental pode ser bastante útil quando não se tem muito acesso a membros do governo ou dados oficiais. Em estudo no Paquistão, Islam (2004) utilizou documentos oficiais de jornais e revistas para relacionar fenômenos como a corrupção, o nepotismo e a diferença entre sexos na cultura histórica daquele país.

Os dados secundários mais referenciados nos estudos que realizaram pesquisa documental, considerando toda amostra da pesquisa, foram os seguintes: (a) Published Documents, Media Reports; (b) Official Documents, Newspaper Reports; e (c) Police Documents, unpublished documents (Gong \& Zhou, 2015; Cobarzan, Dragos, \& Neamtu, 2008; Peisakhin \& Pinto, 2010; Peacock \& Cordner, 2016; Tessema, Soeters, \& Ngoma, 2009).

\subsection{Conceitos de corrupção}

Esta seção busca apresentar um panorama do conceito de corrupção, conforme relatado na literatura internacional em estudo. Ou seja, de que maneira o conceito de corrupção vem sendo definido e suas implicações para a prática e para a abordagem científica do tema. É importante observar que práticas como a designação de parentes para exercerem cargos públicos (nepotismo), a troca de favores com fins de se obter benefícios próprios (clientelismo) e a confusão entre o patrimônio público e o privado (patrimonialismo), embora possam gerar fragilidades institucionais que podem favorecer a corrupção, não podem ser confundidos com corrupção propriamente dita, pois tratam de conceitos diferentes, como será observado nas próximas linhas.

No que se refere especificamente ao conceito de corrupção, trata-se de um fenômeno complexo, em relação ao qual qualquer definição será limitada, passível de ambiguidades e dependerá do contexto social em que se pretende defini-lo. A corrupção é um fenômeno em constante mudança, seu conceito é definido de acordo com interesses pessoais, valores culturais e socioeconômicos de determinada sociedade. Pode haver tipos de corrupção que são socialmente aceitos e outros inaceitáveis e, portanto, criminalizados (Roman \& Miller, 2014; Yeboah-Assiamah, Asamoah, Bawole, \& Musah-Surugu, 2016; Graycar, 2015). 
Uma definição que, apesar de ser bastante ampla, encaixa-se em diversos casos e, por isso, oferece uma base comparativa, além de ser independente de definição legal, pode ser representada no seguinte conceito: abuso de poder público em favor de interesse privado para ganhos ilícitos (Rose-Ackerman, 2002; Mashali, 2012; Andersson, 2017; Dincer \& Guinalp, 2012). Esse conceito tem sido utilizado na literatura principalmente para tratar de aspectos políticos nos governos, como, por exemplo, quando ocorrem atos de corrupção para beneficiar partidos políticos. Nesse conceito, em resumo, o que se observa é que a corrupção pública opera na interface entre os setores públicos e privados, em favor de ganhos privados em detrimento do interesse público (Rose-Ackerman, 2002).

As definições que relacionam poder público e ganhos privados abrem o campo de discussão para diferentes tipos de corrupção e, segundo Anderson (2017), fazem a ligação entre os diferentes tipos de corrupção e a definição legal e social da questão. A definição é suficientemente ampla para incluir suborno e favoritismo como abuso de poder público para favorecimento de familiares, amigos ou parceiros, desvios e fraudes, como ganhos privados indevidos de organizações ou de cidadãos por enganar os outros ou desrespeitar as responsabilidades profissionais e conflito de interesse. Para Anderson (2017), esse conceito é suficientemente amplo para permitir a comparação entre diferentes esferas, tais como executivo, legislativo e judiciário, governo federal e governos locais e entre diferentes países.

\subsection{Causas da Corrupção}

A corrupção pode assumir diversas formas e, como observado anteriormente, essas formas também são contextuais. Exemplo disso é o caso do nepotismo, que não é visto como corrupção em alguns países. Todavia, na maioria dos casos, as formas mais comuns de corrupção envolvem aceitar dinheiro e outras recompensas para concessão de benefícios, como, por exemplo, fechamento de contratos; violação de procedimentos formais para promover interesses pessoais; recebimento de propina de empresas, atores privados, outros países ou organismos internacionais; intervenções em processos judiciais com vista a ganhos pessoais; nepotismo; roubo; superfaturamento; cobrança ou não cobrança de tributos de forma ilegal, além de diversos tipos de fraude.

Nesse aspecto, chama atenção o estudo realizado por Mashali (2012), em que a autora debate duas formas de corrupção e suas correlações: "pequena e grande corrupção". Em estudo realizado no Irã, essa mesma autora, por meio da realização de 30 entrevistas e análise quantitativa de dados, observou que, quanto maior a percepção de corrupção em grande escala, maior é a corrupção em pequena escala $(b=0.942 ; t=134.114 ; \mathrm{p}$ $<.001)$. De acordo com a pesquisa, a pequena corrupção envolve a troca de pequenas quantias e favores entre agentes, geralmente envolvendo agentes públicos que não ocupam posições no alto escalão dos governos, como, por exemplo, policiais e burocratas de rua. Por outro lado, a grande corrupção envolve agentes que atuam no alto escalão do governo (Mashali, 2012). O estudo traz outras questões a serem debatidas, tais como: a partir do momento em que a cúpula se contamina pela corrupção, os outros escalões vão se contaminando também? Há um movimento top-down da corrupção? Esse movimento poderia estar sendo formado por redes que se ligam do topo até ao baixo escalão? Obviamente, outros estudos empíricos em outros contextos seriam necessários para responder a essas perguntas.

Já o estudo de Nickson \& Lambert (2002) relata um al to nível de corrupção institucionalizada no Paraguai, cujas causas remontam às reformas dos anos de $1954 \mathrm{e} 1989$, nos quais o governo paraguaio empreendeu um grande processo de abertura econômica do país. Nesse período, segundo os autores, o governo encorajou e foi tolerante com a corrupção, pois essa era a forma de ligar elites e funcionários públicos ao regime político vigente, já que os ganhos dos agentes por meio da corrupção garantiam a lealdade ao regime, ao mesmo tempo que não representavam impacto financeiro no orçamento público daquele país.

As reformas vêm sendo um tema bastante debatido na literatura. As reformas neoliberais têm sido associadas à incidência da corrupção, sobretudo quando se debate o tema privatização. Nesse sentido, 
reformas mais recentes como as ocorridas na Ucrânia, na Geórgia, na China e na Indonésia, são citadas como ponto de partida para o combate e a prevenção da corrupção (Nickson \& Lambert, 2002; Gong T. , 2011; Zhu \& Jiao, 2012; Peacock \& Cordner, 2016; Jun, Wang, \& Wang, 2014). As reformas apresentadas nessas publicações são entendidas de forma ampla e contextual e alguns termos são recorrentes, tais como: reforma da nova administração pública (New Public Management - NPM), reformas neoliberais, reforma política e reforma da polícia (Pillay, 2008; Zhu \& Jiao, 2012; Schütte, 2012; Peacock \& Cordner, 2016). Como exemplo de estudo que relaciona reformas e corrupção, Aziz (1999) investigou a reforma econômica ocorrida na Malásia em 1969. Essa reforma criou dezenas de instituições públicas, porém a ausência de um mecanismo central de coordenação para essas instituições, além de criar amplos poderes discricionários, fez com que a corrupção se espalhasse no país (Aziz, 1999).

Em grande parte dos estudos revisados o foco dos autores não necessariamente está relacionado à questão e/ou ao objeto da reforma, mas sim às mudanças institucionais causadas e seus mecanismos relacionados ao combate, prevenção ou à tolerância da corrupção, independente de qual seja a reforma e seus propósitos. A discussão a respeito das mudanças e as maneiras como as reformas lidaram com a prevenção e o combate à corrupção será novamente abordada no tópico que trata do combate à corrupção.

\subsection{Consequências da corrup̧̧ão}

Além da evasão de receitas tributárias, já mencionada anteriormente, a corrupção pode trazer diversas consequências negativas para os países. Em alguns estudos o desenvolvimento econômico e a corrupção estão relacionados de forma inversamente proporcional (Gong, Wang, \& Ren, 2015; Paolo, 1995; Dincer \& Guinalp, 2012), ou seja, quanto mais corrupção, menos desenvolvimento. Aparentemente os efeitos da corrupção no desenvolvimento econômico parecem ser bastante profundos (Villoria, Ryzin, \& Lavena, 2013).

Diversos estudos mencionam ou tratam do paradoxo da corrupção e o desenvolvimento econômico dos países (Paolo, 1995; Yang, 2009; Andrei, Matei, Tuşa, \& Nedelcu, 2009). Para Yang (2009), por exemplo, um dos grandes desafios da China em sua transição para uma economia de mercado é o conflito existente entre o desenvolvimento econômico e a luta contra a corrupção. No mesmo sentido, um desafio enfrentado pela Coreia do Sul é responder aos problemas que envolvem o desenvolvimento econômico sustentável em nível local e o combate à corrupção política (Kim, 2010). Em artigo que trata dos desafios e oportunidades do combate à corrupção na Nigéria, Idemudia, Gragg e Best (2010) afirmam que o suborno e a corrupção em larga escala aumentam os custos e os riscos de se fazer negócios, enfraquecendo as normas sociais, dificultando a cooperação, minando as instituições e, consequentemente, gerando um desenvolvimento econômico deficiente. Esses mesmos autores tratam da região do delta na Nigéria, uma região rica em produção de petróleo. É importante notar que não foi encontrado nenhum estudo empírico capaz de identificar quais seriam os mecanismos que levariam ao desequilíbrio econômico.

Todavia, a discussão a respeito da relação entre o desenvolvimento econômico e a corrupção vai além do paradoxo aparente. Rose-Ackerman (2002) afirma que em alguns países, como Indonésia, Tailândia e Coreia do Sul, a corrupção e o crescimento econômico caminharam lado a lado. Segundo Erlingsson, Bergh e Sjölin (2008), apesar de estudos mostrarem um efeito negativo da corrupção no desenvolvimento econômico e uma consequente desaceleração no grau de modernização dos países, em alguns casos a corrupção pode explicar o aumento nos níveis de modernização dos países, sobretudo em países pobres. Nesse sentido, de maneira surpreendente, a corrupção tem sido observada de maneira positiva. Os argumentos giram em torno da capacidade de a corrupção 'lubrificar' as burocracias, pois, considerando que as regras serão corrompidas, alocar recursos de maneira mais eficiente depende de práticas de corrupção, uma vez que muitos empreendimentos não sairiam do papel se não fosse por essa via (Rose-Ackerman, 2002; Gong, Wang, \& Ren, 2015; Walton, 2013). 
Por outro lado, se as pessoas passam a ver o lado positivo da corrupção, podem passar a adotar atitudes mais tolerantes em relação a determinadas práticas, como, por exemplo, o suborno. Assim, as pessoas podem entender que essas práticas não são prejudiciais para o interesse público, uma vez que elas passam a justificar o uso de meios ilícitos como uma forma de lidar com a arbitrariedade das regras e as regulações governamentais (Gong, Wang, \& Ren, 2015). No estudo de Gong, Wang e Ren (2015), os autores sustentam a hipótese de que quanto mais as pessoas veem os benefícios da corrupção, mais tolerantes elas se tornam e, consequentemente, mais resistentes para lutar contra a corrupção. Tais estudos podem levantar debates ainda não resolvidos, tais como a avaliação do custo benefício em se combater a corrupção e se de fato a corrupção pode trazer algum benefício para governos e sociedades.

Apesar de não se poder comprovar que a corrupção traga algum tipo de benefício para governos e sociedades, os argumentos contrários parecem ser significativamente relevantes no que se refere aos efeitos da corrupção. Mesmo que a corrupção seja benéfica, até determinado ponto a sua tolerância ao longo do tempo pode levar à captura do Estado e os desvios acabam superando os supostos benefícios. Todavia, é importante ressaltar que a eficiência econômica não é a única meta a ser almejada pelos países. A corrupção pode gerar distorções na distribuição de renda, minar a estabilidade política de um país e até mesmo afastar investimentos externos e interno. A corrupção também pode comprometer a alocação de recursos públicos e gerar descrédito da população em relação à atividade política do país (Rose-Ackerman, 2002; Cordis \& Milyo, 2016; Villoria, Ryzin, \& Lavena, 2013)

\subsection{Formas de medir a corrup̧ão}

Como já apresentado, os indicadores mais utilizados de corrupção são o Transparency International's Corruption Perceptions Index (CPI), da organização Transparência Internacional e o The World Bank's Worldwide Governance Indicators, do Banco Mundial. As medidas comparativas de corrupção, sobretudo internacionais, enfrentam problemas técnicos já bem conhecidos, principalmente em relação aos critérios de validade científica. Alguns índices não consideram, por exemplo, o suborno como manifestação da corrupção. Além disso, de acordo com o conceito de corrupção adotado, algumas atividades exercidas por membros do Poder Legislativo podem não ser consideradas ilícitas, enquanto no Poder Executivo as mesmas atividades podem ser consideradas corrupção.

Os cidadãos de países diferentes podem ter diferentes visões a respeito do fenômeno da corrupção (Andersson, 2017). Em estudos qualitativos, o entrevistado pode relacionar a corrupção aos mais diversos conceitos, distorcendo, assim, os resultados dos estudos, ou associando o que é ou o que não é corrupção ao contexto de seu país, conforme será discutido posteriormente. Dessa forma, os resultados se baseiam em julgamentos subjetivos que retratam a percepção do cidadão a respeito da corrupção em determinado contexto (Rose-Ackerman, 2002; Andersson, 2017). Em resumo, pode-se dizer que não existem dados muito confiáveis em relação à amplitude da corrupção em países diferentes.

Em estudo conduzido em duas cidades da China, Gong, Wang, \& Ren (2015) encontraram significativas diferenças na interpretação do termo corrupção entre os entrevistados de Hong Kong e os entrevistados da China Continental. Em uma escala de 0-12, em média, os entrevistados de Hong Kong tiveram mais facilidade em identificar a corrupção com uma forma de abuso de poder público $(\mathrm{x}=10.5 ; \mathrm{SD}=1.8)$ do que identificar a corrupção como um desvio de ética no setor privado $(x=8 ; S D=2.1)$. Já na China Continental, segundo os autores, os entrevistados apresentaram uma visão muito mais ampla de corrupção e associaramna de maneira mais significativa a abusos de profissionais privados $(\mathrm{x}=10.1 ; \mathrm{SD}=1.9)$ do que ao abuso de poder público $(\mathrm{x}=9.1, \mathrm{SD}=2.4)$. Para os autores, essas diferenças podem estar associadas à interpretação que os moradores de Hong Kong dão ao suborno e ao abuso de poder público, em decorrência da legislação vigente e do programa de conscientização contra a corrupção. Por outro lado, a corrupção é menos definida na China Continental, pois abrange não somente a violação de regras oficiais, mas também a má conduta 
moral (Gong, Wang, \& Ren, 2015). Esse estudo mostra a dificuldade de se empreender estudos comparativos considerando a percepção dos entrevistados.

O nível de análise também é um problema a ser considerado, pois ele pode variar de acordo com o conceito de corrupção adotado. Por exemplo, algumas atividades exercidas por membros do Poder Legislativo podem não ser consideradas ilícitas, enquanto no Poder Executivo de um mesmo país as mesmas atividades podem ser consideradas ilícitas. Quando se trata dos índices citados, diversos atores são considerados em nível nacional, como partidos políticos, parlamentares, atores do setor privado, mídia, educação, religião, autoridades fiscais e financeiras, polícia, órgãos de registro e permissões e o Poder Judiciário. Porém, segundo Andersson (2017), esses índices não consideram a distribuição geográfica em nível local. Esse mesmo autor mostra que na Suécia os dados a respeito da corrupção nos governos federal, estadual e local são bem diferentes quando comparados aos dados globais, pois a maioria dos índices de corrupção não define o que seria e o que não seria corrupção.

Como se observa, as tentativas de medir a corrupção na Administração Pública encontram diversos desafios metodológicos, sobretudo porque é difícil medir algo que não pode ser observado diretamente (Graycar, 2015; Pashev, 2011). Além disso, em muitos países, há dificuldades em se conseguir relatórios oficiais e dados públicos. Por isso, uma das formas de se realizar estudos comparativos entre países é criar índices subjetivos (Cordis \& Milyo, 2016).

Outros indicadores tentam, além da percepção, identificar evidências de corrupção ou de fragilidades institucionais que podem levar à corrupção. Esses indicadores não estão diretamente ligados a taxas ou índices de corrupção, mas sim a mecanismos de combate à corrupção. Um deles é o Governance Matter, do World Bank Institute, que é um indicador agregado que trata de diversas dimensões de governança, sendo que uma das dimensões consideradas diz respeito aos mecanismos de controle da corrupção. Outro indicador é o Anti-corruption Mechanisms, da Organisation for Economic Co-operation and Development (OECD). O indicador é baseado na busca de evidências sobre os mecanismos de combate à corrupção (Head, 2012). Em um estudo a respeito da melhoria do processo orçamentário no Afeganistão, Gunn e Straussman (2015), apresentam os índices Fragile States Index, do The Fund for Peace (FFP), e o Open Budget Index, do International Budget Partnership (IBP). O primeiro é um índice anual baseado em indicadores de risco, elaborados a partir de diversos relatórios e artigos científicos, e o segundo é um indicador baseado na transparência orçamentária do país, que avalia oito documentos-chave para o orçamento público e se eles estão sendo disponibilizados dentro do prazo e de acordo com as normas internacionais em 115 países do mundo.

Pode-se afirmar que cada índice conta uma história e, dessa forma, os indicadores simplificam conceitos complexos como corrupção e governança. Outras formas de se medir a corrupção vêm sendo abordadas pela literatura, no entanto, esses indicadores alternativos vêm sendo usados com uma abordagem diferente dos principais indicadores apresentados. Geralmente, os estudos com os indicadores alternativos não buscam uma ampla comparação entre países, apenas medir questões pontuais em relação à corrupção. Nesse sentido, tenta-se medir a incidência de corrupção com base em reportagens e notícias em jornais e revistas, relatórios e casos reportados por autoridades e organizações de combate à corrupção e levantamento de campo, inclusive por meio de observação.

Também tem-se tentado medir a corrupção por meio de dados penais obtidos junto às organizações de polícia ou do Judiciário (Dincer \& Guinalp, 2012; Guinn \& Straussman, 2016; Bolcha \& Rovný, 2016; Cordis \& Milyo, 2016). Uma consideração igualmente importante é que os resultados ex-post só mostram os casos em que de fato os corruptos foram presos e não passam uma medida da amplitude da corrupção. Uma das vantagens é que o indicador pode mostrar casos atuais de corrupção, que permitem estudar novos casos e novas formas que a corrupção pode assumir.

Além disso, nem sempre dados oficiais e não publicados estão disponíveis e o governo de muitos países pode se negar a fornecer os dados secundários também. Na República Checa, Bolcha e Rovný (2015) desenvolveram um método para levantamento de dados em campo voltado para identificar níveis de 
corrupção nos registros de carros, uma vez que as autoridades se mostraram relutantes em fornecer os dados oficiais para os pesquisadores. Como recurso à falta de dados oficiais, os pesquisadores foram para uma praça movimentada de Praga e anotaram os dados dos veículos que passavam.

Outro aspecto igualmente relevante é que nem sempre os dados oficiais são confiáveis. Cordis e Milyo (2016) mostram que os dados elaborados pela seção de integridade pública (PIN) do Departamento de Justiça americano, que são amplamente utilizados pela comunidade acadêmica, apresentam diversas fragilidades quando utilizados para medir taxas de corrupção. Entre outros problemas, os dados incluem como corrupção atos de improbidade administrativa que nem sempre são enquadrados na lei como corrupção. Segundo Cordis e Milyo (2016), o registro "corrupção oficial” é a designação para qualquer processo em tribunal federal de funcionários públicos federais, estaduais ou locais por má conduta oficial ou mau uso do cargo, que não necessariamente se enquadram em casos de corrupção.

\subsection{Prevenção e combate à corrupção}

Grade parte dos artigos estudados trata de reformas e combate à corrupção, enquanto outros salientam o conceito de prevenção da corrupção e não seu combate. Ao que parece, são abordagens complementares e que fazem bastante sentido. Esta seção aborda dois dos principais debates a respeito da prevenção e do combate à corrupção.

Foi observado que as reformas parecem ser a chave para adoção de modelos de combate e prevenção à corrupção (Kickert, 2011). É importante observar que essa perspectiva leva a uma visão pouco incremental do processo, o que pode não ser tão realista. Todavia, não se pode descartar a capacidade de essas reformas gerarem significativas mudanças institucionais no que se refere ao combate, prevenção e/ou tolerância à corrupção. $\mathrm{O}$ que se tem chamado de reformas nessa literatura diz respeito a uma série de movimentos que muitas vezes não estão ligados diretamente à corrupção, tais como as reformas administrativas empreendidas por diversos países na década de 1980 e 1990, conhecidas como reformas neoliberais ou reformas gerenciais, como aquelas ocorridas no Reino Unido, Austrália, Nova Zelândia, Estados Unidos, Vietnã, Grécia, Itália, Portugal, Espanha e em outros países (Gregory, 1999; Therkildsen, 2000; Painter, 2003; Kickert, 2011).

Outros tipos de reformas também são importantes na prevenção e combate à corrupção, como as reformas do sistema judicial na Bulgária (Frison-Roche \& Sodev, 2005), a reforma da polícia na Ucrânia (Peacock \& Cordner, 2016) e a reforma no sistema de compras da Turquia (Ayhan \& Üstüner, 2015). Outros tipos de reformas igualmente referenciadas na literatura dizem respeito ao combate e prevenção da corrupção, denominadas de Anti-Corruption Reforms (Schütte, 2012; Gong, Wang, \& Ren, 2015; Gillespie, Girgis, \& Mayer, 1996). Especificamente quanto à recente reforma da Ucrânia (2014), dois mecanismos de pressão podem ser observados para que ações de combate à corrupção fossem implementadas. Primeiro, a necessidade de aumentar as parcerias comerciais com os países da Europa e segundo, o empoderamento dos ativistas para exercer o controle externo das organizações (watchdogs). As reformas anticorrupção promovidas em Gana, em 1986, também foram associadas ao aumento de receita e redução da corrupção, com esse aumento relacionado à redução da evasão tributária, como já discutido anteriormente (Joshi \& Ayee, 2009).

O termo watchdog ("cão de guarda" em inglês) tem sido usado para denominar diversos atores ligados ao combate à corrupção. Exemplos de watchdogs são as agências de combate à corrupção, organizações não governamentais (como, por exemplo, as Audit Initiative, no Vietnã), ouvidorias, ativistas e outros membros da sociedade, órgãos de controle, judiciário, agências reguladoras, e a mídia (Gong T. , 2009; Jiménez \& Quesada, 2012; Jancsics \& Jávor, 2012; Vian, Brinkerhoff, Feeley, Salomon, \& Vien, 2012; Peacock \& Cordner, 2016). Especificamente quanto às agências governamentais de combate à corrupção, é possível encontrar diversos tipos de agências, com diversos modelos de autonomia, modo de operar e papéis no combate à corrupção. 
Como exemplo de agências que atuam como watchdogs, pode-se citar os casos da Tanzânia, Austrália e China. Na Tanzânia, foi criada a Commission on the Causes of Corruption, uma agência que realiza diversos estudos no sentido de se identificar as prováveis causas da corrupção. Na Austrália, foi criada a Government Investigations and Compliance, que opera na forma de um watchdog em nível local (Fjeldstad, 2003; Masters \& Graycar, 2016). No caso da China, especificamente em Hong Kong, foi criada a Independent Commission against Corruption (ICAC), à qual a literatura do país credita considerável sucesso no controle da corrupção (Gong, Wang, \& Ren, 2015; Doig, Watt, \& Williams, 2006). Na China Continental, a primeira agência data de 1980, a Discipline Inspection Committees of the Chinese Communist Party. Interessante notar que na China a postura das agências de combate à corrupção têm priorizado a atuação preventiva em vez das ações repressivas. Como resultado disso, os termos relacionados ao "combate à corrupção" foram mudados para "prevenção" e "anticorrupção" (GONG, 2011). Como se observa, existem diversos tipos de agências e funções para essas agências no combate à corrupção.

Além das agências, outro mecanismo significativamente importante para o combate à corrupção é a transparência. Em estudo a respeito do Afeganistão, Gunn e Straussman (2015) descrevem como os investidores/doadores forçam o governo a adotar medidas de transparência que, consequentemente, contribuem para lidar com a corrupção. Uma contribuição interessante que esse artigo traz para o debate é a relação da dependência orçamentária e o grau de transparência. Segundo esses mesmos autores, embora existam estudos relacionando inversamente dependência orçamentária e transparência financeira, acreditase que, no caso do Afeganistão, a relação observada é diretamente proporcional, e o que faz com que os indicadores de transparência melhorem é a pressão imposta por investidores e doadores internacionais.

\section{Considerações Finais}

Considerando a proposta deste estudo, pode-se afirmar que os objetivos foram atingidos ao realizar-se o mapeamento da literatura internacional em língua inglesa sobre o tema, como a agenda de pesquisa está sendo direcionada dentro dessa literatura e os principais temas abordados nesses estudos. Nesse sentido, pode-se observar que os resultados encontrados apontam para uma agenda de pesquisa que gira em torno de alguns tópicos centrais. Observou-se ainda que a produção sobre corrupção vem crescendo dentro do campo da Administração Pública. Estudos comparativos e em nível local têm sido a tendência. Questões como as causas, consequências, formas de se medir e de se combater e o próprio conceito de corrupção envolvem grande parte das discussões sobre a temática. Outras questões interessantes ainda estão em aberto e oferecem uma boa gama de temas de pesquisa, tais como os possíveis benefícios da corrupção, formas de se identificar e medir a corrupção, os efeitos das recentes reformas contra a corrupção no mundo e a transparência como medida adotada nos distintos países.

Vale ainda aprofundar pesquisas sobre corrupção no Brasil, haja vista a especificidade do fenômeno em cada localidade, independente da produção científica sobre o tema, mas a partir de pesquisa documental, também porque o Estado Brasileiro vem adotando medidas de prevenção e combate à corrupção como signatário da Convenção das Nações Unidas contra a Corrupção desde 2003 e participado de fóruns internacionais sobre o tema, a exemplo do Seminar on International Cooperation in Civil and Administrative Proceedings Relating to Corruption In The G20, realizado em Brasília me abril de 2017.

\section{REFERÊNCIAS}

Andersson, S. (2017). Beyond Unidimensional Measurement of Corruption. Public Integrity, 19, 58-76. doi:10.1080/10999922.2016.1200408

Andrei, T., Matei, A., Tuşa, E., \& Nedelcu, M. (2009). Characteristics of the reforming process in the Romanian public administration system. Transylvanian Review of Administrative Sciences, 13-31. 
Fábio Jacinto Barreto de Souza, et al. Corrupção no Setor Público: Agenda de Pesquisa e Principais...

Ayhan, B., \& Üstüner, Y. (sep de 2015). Governance in public procurement: the reform of Turkeyś public procurement system. International Review of Administrative Sciences, 81, 640-662. doi: $10.1177 / 0020852314548153$

Aziz, T. A. (1999). Malaysia Incorporated: Ethics on Trial. Australian Journal of Public Administration, 58, 19-25. doi: $10.1111 / 1467-8500.00123$

Bolcha, P., \& Rovný, J. (2016). Luck or luxury? Possible corruption in the car registration process in the Czech Republic. Journal of Public Policy, 36, 603-638. doi:10.1017/S0143814X15000276

Chan, H. S., \& Ma, J. (2011). How are they paid? A study of civil service pay in China. International Review of Administrative Sciences, 77, 294-321. doi:10.1177/0020852311399231

Chang, E. C., \& Kerr, N. N. (2017). An Insider-Outsider Theory of Popular Tolerance for Corrupt Politicians. Governance, 30, 67-84. doi:10.1111/gove.12193

Chen, Z., Li, D., \& Wang, J. (2010). Citizens \#Attitudes Toward Local Government Public Services. Public Performance $\{\backslash \&\}$ Management Review, 34, 221-235. doi:10.2753/PMR1530-9576340204

Cheung, A. B. (2012). One country, two experiences: administrative reforms in China and Hong Kong. International Review of Administrative Sciences, 78, 261-283. doi:10.1177/0020852312438044

Cobarzan, B. V., Dragos, D. C., \& Neamtu, B. (2008). Free Access To Public Information: Enforcement, Appeals and Judicial Review. a Comparative Perspective From Cee Countries. Transylvanian Review of Administrative Sciences, 53-63.

Cordis, A. S., \& Milyo, J. (2016). Measuring Public Corruption in the United States: Evidence From Administrative Records of Federal Prosecutions. Public Integrity, 18, 127-148. doi:10.1080/10999922.2015.1111748

Deng, X., Wang, Y., Zhang, Q., Huang, J. X., \& Cui, J. (2014). Analysis of fraud risk in public construction projects in China. Public Money \& Management, 34, 51-58. doi:10.1080/09540962.2014.865939

Dincer, O., \& Guinalp, B. (2012). Corruption and Income Inequality in the United States. Contemporary Economic Policy, 30, 283-292. doi:10.1111/j.1465-7287.2011.00262.x

Doig, A., Watt, D., \& Williams, R. (2006). Hands-on or hands-off? Anti-corruption agencies in action, donor expectations, and a good enough reality. Public Administration and Development, 26, 163-172. doi:10.1002/ pad.398

Doig, A., Watt, D., \& Williams, R. (2007). Why do developing country anti-corruption commissions fail to deal with corruption? Understanding the three dilemmas of organisational development, performance expectation, and donor and government cycles. Public Administration and Development, 27, 251-259. doi:10.1002/pad.452

Erlingsson, G. O., Bergh, A., \& Sjölin, M. (2008). Public Corruption in Swedish Municipalities - Trouble Looming on the Horizon? Local Government Studies, 34, 595-608. doi:10.1080/03003930802413780

Esposito, P., \& Ricci, P. (2015). How to turn public (dis)value into new public value? Evidence from Italy. Public Money \& Management, 35, 227-231. doi:10.1080/09540962.2015.1027499

Fjeldstad, O.-h. (may de 2003). Fighting fiscal corruption: lessons from the Tanzania Revenue Authority. Public Administration and Development, 23, 165-175. doi:10.1002/pad.278

Forgia, G., Raha, S., Shaik, S., Maheshwari, \& Ali, R. (2015). Parallel System Human REsource Management in India 's Public Health Services: A View from the Front Lines. Public Administration, pp. 372-389.

Friese, S. (2014). Qualitative Data Analysis. USA: SAGE Publications.

Frison-Roche, F., \& Sodev, S.-D. (2005). The issues involved in the reform of the Bulgarian judicial system. International Review of Administrative Sciences, 71, 593-606. doi:10.1177/0020852305059600

Gillespie, P., Girgis, M., \& Mayer, P. (1996). "This great evil": anticipating political obstacles to development. Public Administration and Development, 16, 431-453. doi:10.1002/(SICI)1099-162X(199612)16:5<431::AIDPAD893>3.3.CO;2-F

Gong, T. (2009). Audit for Accountability in China: An Incomplete Mission. Australian Journal of Public Administration, 68, S5--S16. doi:10.1111/j.1467-8500.2009.00617.x 
Gong, T. (2011). An ínstitutional turn \#in integrity management in China. International Review of Administrative Sciences, 77, 671-686. doi:10.1177/0020852311419391

Gong, T., \& Wu, A. M. (2012). Does Increased Civil Service Pay Deter Corruption? Evidence from China. Review of Public Personnel Administration, 32, 192-204. doi:10.1177/0734371X12438247

Gong, T., \& Zhou, N. (2015). Corruption and marketization: Formal and informal rules in Chinese public procurement. Regulation \& Governance, 9, 63-76. doi:10.1111/rego.12054

Gong, T., Wang, S., \& Ren, J. (2015). Corruption in the Eye of the Beholder: Survey Evidence from Mainland China and Hong Kong. International Public Management Journal, 18, 458-482. doi:10.1080/10967494.2015.1057629

Graycar, A. (2015). Corruption: Classification and analysis. Policy and Society, 34, 87-96. doi:10.1016/ j.polsoc.2015.04.001

Gregory, R. J. (1999). Social Capital Theory and Administrative Reform: Maintaining Ethical Probity in Public Service. Public Administration Review, 59, 63. doi:10.2307/977480

Grimes, M., \& Wangnerud, L. (2010). Curbing Corruption Through Social Welfare Reform? The Effects of Mexicoś Conditional Cash Transfer Program on Good Government. The American Review of Public Administration, 40, 671-690. doi:10.1177/0275074009359025

Grodeland, A., Koshechkina, T., \& Miller, W. L. (1997). Alternative strategies for coping with officials in different postcommunist regimes: the wormś eye view. Public Administration and Development, 17, 511-528. doi:10.1002/(sici)1099-162x(199712)17:5<511::aid-pad983>3.3.co;2-8

Guinn, D. E., \& Straussman, J. D. (mar de 2016). Improving the Budget Process in Fragile and Conflict-Ridden States: Two Modest Lessons from Afghanistan. Public Administration Review, 76, 263-272. doi:10.1111/puar.12397

Harbers, I. (2015). Taxation and the Unequal Reach of the State: Mapping State Capacity in Ecuador. Governance, 28, 373-391. doi:10.1111/gove.12117

Head, B. W. (2012). The contribution of integrity agencies to good governance. Policy Studies, 33, 7-20. doi:10.1080/01442872.2011.601200

Idemudia, U., Cragg, W., \& Best, B. (2010). The challenges and opportunities of implementing the integrity pact as a strategy for combating corruption in Nigeriaś oil rich Niger Delta region. Public Administration and Development, 30, 277-290. doi:10.1002/pad.576

Islam, N. (2004). Sifarish, Sycophants, Power and Collectivism: Administrative Culture in Pakistan. International Review of Administrative Sciences, 70, 311-330. doi:10.1177/0020852304044259

Jancsics, D., \& Jávor, I. (2012). Corrupt Governmental Networks. International Public Management Journal, 15, 62-99. doi:10.1080/10967494.2012.684019

Jiménez, F., \& Quesada, M. (2012). Badly Designed Institutions, Informal Rules and Perverse Incentives : Local Government Corruption in Spain. Lex Localis - Journal of Local Self-Government, 10, 363-381. Fonte: http:// pub.lex-localis.info

Johnston, M. (2012). Corruption control in the United States: law, values, and the political foundations of reform. International Review of Administrative Sciences, 78, 329-345. doi:10.1177/0020852312438782

Joshi, A., \& Ayee, J. (2009). Autonomy or organisation? Reforms in the Ghanaian internal revenue service. Public Administration and Development, 29, 289-302. doi:10.1002/pad.535

Jun, K.-N., Wang, F., \& Wang, D. (2014). E-Government Use and Perceived Government Transparency and Service Capacity. Public Performance $\{\backslash \&\}$ Management Review, 38, 125-151. doi:10.2753/PMR1530-9576380106

Ke, Y., Wang, S., \& Chan, A. P. (2013). Risk Misallocation in Public-Private Partnership Projects in China. International Public Management Journal, 16, 438-460. doi:10.1080/10967494.2013.825508

Kickert, W. (2011). Distinctiveness Of Administrative Reform In Greece, Italy, Portugal And Spain. Common Characteristics Of Context, Administrations And Reforms. Public Administration, 89, 801-818. doi:10.1111/ j.1467-9299.2010.01862.x 
Kim, S. (2010). Public Trust in Government in Japan and South Korea: Does the Rise of Critical Citizens Matter? Public Administration Review, 70, 801-810. doi:10.1111/j.1540-6210.2010.02207.x

Kopytko, N. (2016). Change and transition: the climate of Ukraineś agri-food sector. Climate Policy, 16, 68-87. doi:10.1080/14693062.2014.979131

Li, H., Xiao, H., \& Gong, T. (2015). The impact of economic well-being on perceptions of anti-corruption performance: Evidence from China. Policy and Society, 34, 97-109. doi:10.1016/j.polsoc.2015.05.001

Lin, J. Y. (1995). CAN CHINAŚ â\&128;\&156;MINI-BANGâ\&128;\&157; SUCCEED? Contemporary Economic Policy, 13, 10-14. doi:10.1111/j.1465-7287.1995.tb00706.x

Mashali, B. (2012). Analyzing the relationship between perceived grand corruption and petty corruption in developing countries: case study of Iran. International Review of Administrative Sciences , 78 , 775-787. doi: $10.1177 / 0020852312455991$

Masters, A. B., \& Graycar, A. (2016). Making Corruption Disappear in Local Government. Public Intergrity, 18, 42-58. doi:10.1080/10999922.2015.1093400

Nickson, A., \& Lambert, P. (2002). State reform and the privatized state in Paraguay. Public Administration and Development, 22, 163-174. doi:10.1002/pad.218

Painter, M. (2003). Public administration reform in Vietnam: problems and prospects. Public Administration and Development, 23, 259-271. doi:10.1002/pad.249

Painter, M. (2008). From Command Economy to Hollow State? Decentralisation in Vietnam and China. Australian Journal of Public Administration, 67, 79-88. doi:10.1111/j.1467-8500.2007.00570.x

Paolo, M. (1995). Corruption and Growth. The Quarterly Journal of Economics, 110, pp. 681-712.

Pashev, K. V. (2011). Corruption and Accession. Public Management Review, 13, 409-432. doi:10.1080/14719037.2011.553270

Peacock, R., \& Cordner, G. (2016). “Shock Therapy” in Ukraine: A Radical Approach to Post-Soviet Police Reform. Public Administration and Development, 36, 80-92. doi:10.1002/pad.1748

Peisakhin, L., \& Pinto, P. (2010). Is transparency an effective anti-corruption strategy? Evidence from a field experiment in India. Regulation \& Governance, 4, 261-280. doi:10.1111/j.1748-5991.2010.01081.x

Pillay, S. (2008). A cultural ecology of New Public Management. International Review of Administrative Sciences, 74, 373-394. doi:10.1177/0020852308095949

Roman, A. V., \& Miller, H. T. (2014). Building Social Cohesion. Administration $\{\backslash \&\}$ Society, 46, 775-795. doi: $10.1177 / 0095399712473987$

Rose-Ackerman. (2002). Corruption and Government. New York: Cambridge University Press.

Rosenbloom, D. H., \& Gong, T. (2013). Coproducing "Clean" Collaborative Governance. Public Performance $\{\backslash \&\}$ Management Review, 36, 544-571. doi:10.2753/PMR1530-9576360403

Schütte, S. A. (2012). Against the ODDS: Anti_Corruption Reform in Indonesia. Public Administration and Development, 32, pp. 38-48.

Škrbec, J., \& Dobovšek, B. (2013). Corruption capture of local self-governments in Slovenia. Lex Localis, 11, 615-630. doi:10.4335/11.3.615-630

Tessema, M. T., Soeters, J. L., \& Ngoma, A. (2009). Decentralization of HR Functions: Lessons From the Singapore Civil Service. Review of Public Personnel Administration, 29, 168-188. doi:10.1177/0734371X09332542

Therkildsen, O. (2000). Public sector reform in a poor, aid-dependent country, Tanzania. Public Administration and Development, 20, 61-71. doi:10.1002/1099-162X(200002)20:1<61::AID-PAD101>3.0.CO;2-T

Tsao, K. K., \& Worthley, J. A. (1996). China: Administrative Corruption - Experience in a Comparative Context. Australian Journal of Public Administration, 55, 22-29. doi:10.1111/j.1467-8500.1996.tb02555.x

Vian, T., Brinkerhoff, D., Feeley, F., Salomon, M., \& Vien, N. (2012). Confronting Corruption in the Health Sector in Vietnam. Public Administration and Development, 32, pp. 49-63. 
Villoria, M., Ryzin, V. a., \& Lavena, C. F. (2013). Social and Political Consequences of Administrative Corruption: A Study of Public Perceptions in Spain. Public Administration Review, 73, 85-94. doi:10.1111/ j.1540-6210.2012.02613.x

Walker, R. M., Brewer, G. a., Bozeman, B., Moon, M. J., \& Wu, J. (2013). An Experimental Assessment of Public Ownership and Performance. Public Management Review, 15, 1208-1228. doi: $10.1080 / 14719037.2013 .825480$

Walton, G. (2013). Is All Corruption Dysfuntional? Perceptions of Corruptio and Ints Consequences in Papua New Guinea. Public Administration and Development, pp. 175-190.

Yang, K. (2009). Institutional Congruence, Ideas, and Anticorruption Policy: The Case of China and the United States. Public Administration Review, 69, S142--S150. doi:10.1111/j.1540-6210.2009.02102.x

Yeboah-Assiamah, E., Asamoah, K., Bawole, J. N., \& Musah-Surugu, I. J. (2016). A socio-cultural approach to public sector corruption in Africa: key pointers for reflection. Journal of Public Affairs, 16, 279-293. doi:10.1002/ pa. 1587

Zhu, X., \& Jiao, Q. (2012). "New Public Management" in China at the Local Level: Competition-Driven Local Public Service Reform in Tianjin. Lex Localis - Journal of Local Self-Government, 10, 153-170. doi:10.4335/10.2.153-170(2012)ISSN

\section{BY-NC-ND}

